Adhesion promotes phase separation in mixed-lipid membranes

This article has been downloaded from IOPscience. Please scroll down to see the full text article.

2008 EPL 8448003

(http://iopscience.iop.org/0295-5075/84/4/48003)

View the table of contents for this issue, or go to the journal homepage for more

Download details:

IP Address: 146.6.180.235

The article was downloaded on 26/10/2010 at 16:05

Please note that terms and conditions apply. 


\title{
Adhesion promotes phase separation in mixed-lipid membranes
}

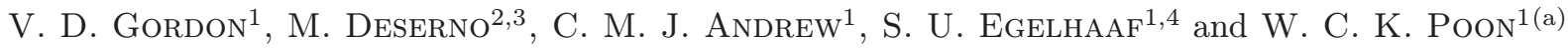 \\ ${ }^{1}$ SUPA, School of Physics, and COSMIC, The University of Edinburgh, James Clerk Maxwell Building, \\ Kings Buildings - Mayfield Road, Edinburgh, EH9 3JZ, Scotland, UK, EU \\ ${ }^{2}$ Max-Planck-Institut für Polymerforschung - Ackermannweg 10, 55128 Mainz, Germany, EU \\ ${ }^{3}$ Department of Physics, Carnegie Mellon University - Pittsburgh, PA 15213-3890, USA \\ ${ }^{4}$ Condensed Matter Physics Laboratory, Heinrich-Heine-University - D-40225 Düsseldorf, Germany, EU
}

received 27 June 2008; accepted in final form 7 October 2008 published online 12 November 2008

PACS 87.16.D- - Membranes, bilayers, and vesicles

PACS 64.75.St - Phase separation and segregation in thin films

PACS 87.17.Rt - Cell adhesion and cell mechanics

\begin{abstract}
We investigate the interplay of domain formation and adhesion in mixed-lipid membranes. Giant unilamellar vesicles consisting of two- and three-component lipid mixtures are studied using confocal fluorescence microscopy. Upon driving the system towards the demixing transition, phase separation is invariably found to occur first in regions where membranes adhere to one another, despite identical lipid headgroups and negligible curvature effects. We propose a simple generic mechanism based on the suppression of thermal shape fluctuations to explain these observations. Our findings suggest novel possibilities by which biomembranes can create and utilize lateral lipid heterogeneities.
\end{abstract}

Copyright (c) EPLA, 2008

Introduction. - Lateral heterogeneities in cell membrane composition were first described a decade ago [1,2]. These suggest a functional reason for the compositional complexity of biological membranes and have prompted a great deal of cross-disciplinary research on mixed lipid bilayers. In particular, biological physicists have focused on simple model systems, such as giant unilamellar vesicles (GUVs), and mapped the phase diagrams of well-characterized binary and ternary lipid mixtures. While being compositionally homogeneous and fluid at sufficiently high temperatures (for which membranes are in the $\mathrm{L}_{\alpha}$ phase), these systems phase segregate upon cooling. Typically, binary mixtures undergo fluid-solid demixing, identifiable for instance by the non-trivial shapes of the emerging solid-like (or "gel") domains [3-5], whose shapes are controlled by the symmetry of lipid packing [6]. Ternary mixtures can also display coexistence between a liquid-ordered $\left(l_{\mathrm{o}}\right)$ and a liquid-disordered $\left(l_{\mathrm{d}}\right)$ phase, with domains of the minority phase having wellrounded boundaries and diffusing freely in the majority phase [7-11]. At a particular (temperature-dependent) composition this fluid-fluid coexistence becomes critical. The concomitant large fluctuations may [12] be connected to the aforementioned inhomogeneities in cellular plasma membranes, known as "lipid rafts" $[1,2,13]$.

\footnotetext{
(a) E-mail: w.poon@ed.ac.uk
}

Isolated lipid bilayers are a convenient and useful idealization, but in many experimental and almost all biological situations membranes adhere to something else - such as other membranes, the cytoskeleton, extra-cellular matrix (ECM), or simply a substrate on which a cell rests [14]. In fact, proper adhesion in such cases is crucial to mechanotransduction, signaling, or cell differentiation and development [15]. Moreover, controlling adhesion to synthetic substrates is crucial for "cell-on-a-chip" technologies [16].

Recent biophysical studies have largely focused on adhesion caused by specific interactions between particular membrane-bound "binder proteins", e.g. in immunological synapses [17] or focal adhesion sites [18]. Experimental work has investigated how model ligand-receptor pairs mediate specific binding [19-21], while theoretical studies have examined the role of binder diffusion [22] and adhesion-dependent enrichment [23,24]. What has remained largely unexplored, however, is the idea that adhesion per se, whether caused by specific or non-specific effects, may perturb a multicomponent mixture enough to trigger non-trivial effects.

In this letter, we demonstrate experimentally that adhesion can induce phase segregation in a multicomponent lipid bilayer, in the absence of any specific proteins or even lipid-lipid interactions. We argue theoretically that such adhesion-induced phase separation is generic, and depends only on the two phases being elastically distinct. 
Table 1: Lipid mixtures used in this study. The last column indicates the type of ordered phase after demixing.

\begin{tabular}{cccc}
\hline$\#$ & Mixture & Composition & Ordered phase \\
\hline 1 & DOPC:BSM:Chol & $40: 40: 20$ & $\mathrm{l}_{\mathrm{o}}$ \\
2 & DLPC:DSPC & $50: 50$ & $\mathrm{P}_{\beta^{\prime}}$ II \\
3 & DMPC:DSPC & $50: 50$ & $\mathrm{P}_{\beta^{\prime}} \mathrm{II}$ \\
4 & DLPC:DAPC & $50: 50$ & $\mathrm{P}_{\beta^{\prime}} \mathrm{II}$ \\
5 & DLPC:DPPC & $50: 50$ & $\mathrm{P}_{\beta^{\prime}}$ \\
\hline
\end{tabular}

The total free energy contains an entropic term due to shape fluctuations, so that adhesion and demixing into elastically distinct phases are coupled because they both modify the fluctuation spectrum. Calculations using typical parameters show that the coupling is strong enough to cause a notable shift in the demixing temperature of adhered vs. non-adhered membranes. We propose a number of biological situations in which such adhesion-induced phase separation may be important.

Materials and methods. - Membranes in which fluid $l_{\mathrm{o}}$ and $l_{\mathrm{d}}$ phases coexist are made from $\mathrm{DOPC}^{1}$, porcine brain sphingomyelin (BSM), and cholesterol (Chol) in a molar ratio 40:40:20 [25]. To prepare membranes in which fluid and solid phases coexist, equimolar binary mixtures from the lipids DLPC, DMPC, DPPC, DSPC, and DAPC were used (table 1).

GUVs (diameter 10-50 $\mu \mathrm{m}$ ) were prepared using electroformation $[5,7,26,27]$. The chamber was resistively heated and temperature was measured using a thermocouple. Vesicles were formed above the chain-melting temperature for the higher-melting component in each mixture. The temperature was then lowered at $0.1-0.2{ }^{\circ} \mathrm{C} / \mathrm{min}$, resulting in phase separation.

Vesicles were observed in situ while still adhering to each other and to the electrodes on which they formed. For the DLPC: DPPC mixture only, vesicles were electroformed in 100 or $200 \mathrm{mM}$ sucrose solution in a chamber adjusted to allow slight evaporation of the extra-vesicle solution; osmotic pressure then deflated the vesicles and the adherent area visibly increased.

Lipid phase separation was visualized using trace amounts (total: $0.1-0.5 \mathrm{~mol} \%$ ) of the preferentially partitioning amphiphilic fluorescent dyes BODIPY and DiI-C-18 $[4,5]^{2}$. They were both included in all membranes

\footnotetext{
1 "DXPC" stands for the lipid 1,2-Di- $X$-sn-glycero-3phosphatidylcholine, and " $X$ " denotes the two identical fatty acid tails according to: "lauroyl", "myristoyl", "palmitoyl", "stearoyl", "arachidoyl" (saturated, 12, 14, 16, 18, 20 carbons in chain, respectively) and "oleoyl" (unsaturated, cis-double bond at position 9, 18 carbons in chain). All lipids were purchased from Avanti Polar Lipids (Alabaster, AL) and used without further purification.

${ }^{2}$ BODIPY:

2-(4,4-difluoro-5,7-dimethyl-4-bora-3a,4a-diaza$s$-indacene-3-pentanoyl)-hexadecanoyl-sn-glycero-3-phosphocholine, DiI-C-18: 1, 1'-dioctadecyl-3, 3, 3', $3^{\prime}$-tetramethylindocarbocyanine perchlorate. Both dyes were purchased from Molecular Probes (Invitrogen Corporation, Carlsbad, CA).
}

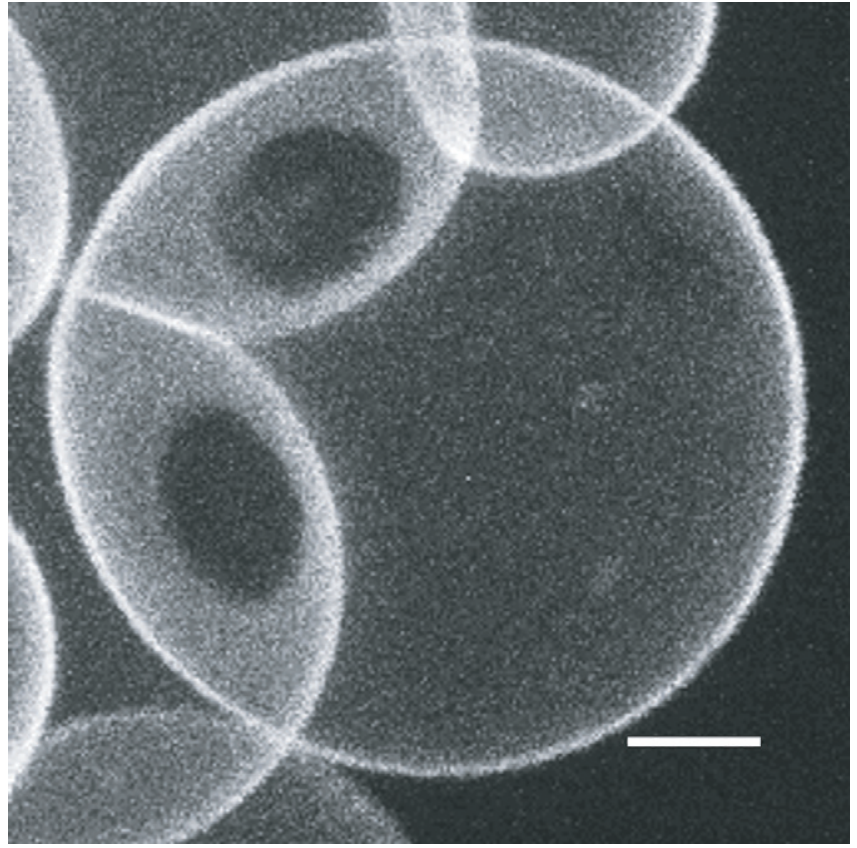

Fig. 1: For mixture 1, liquid-ordered $\left(l_{0}\right)$ domains in a fluid-disordered membrane are first seen in regions where membranes adhere. The $l_{0}$ phase excludes both dyes, DiI-C-18 and BODIPY. Observation temperature: $30^{\circ} \mathrm{C}$. Scale bar: $5 \mu \mathrm{m}$.

and excited at $488 \mathrm{~nm}$. Micron-scale domain formation following demixing can be visualized since fluorophores, distributed homogeneously in single-phase membranes, are preferentially excluded from the phase whose structure is more disrupted by their inclusion $[4-7,9,28]$. Usually this is the more-ordered phase, except for the corrugated ripple phase, $\mathrm{P}_{\beta^{\prime}}$, which accommodates fluorophores with a bulky head group better than, e.g. the flat $\mathrm{L}_{\alpha}$ phase $[4,28]$. Images were acquired using an Nikon TE300 inverted microscope and a BIORAD Radiance $2100 \mathrm{MP}$ confocal system. ImageJ is used to reconstruct vesicle hemispheres from confocal slices; typical results are shown in figs. $1-3$.

Results and discussion. - We study the emergence and location of ordered domains upon lipid demixing for five different mixtures and three different types of phasecoexistence scenarios, table 1 . Mixture 1 segregates into two fluid phases; mixtures $2-5$ show fluid-solid coexistence, with the solid phase being $\mathrm{P}_{\beta^{\prime}}$ II [29] in 2-4 and $\mathrm{P}_{\beta^{\prime}}$ [30] in mixture 5 .

At a temperature slightly above the ordering transition, photo-oxidation effectively depletes the ternary mixture 1 of cholesterol, thus promoting phase separation and causing domains of the liquid-ordered $\left(l_{\mathrm{o}}\right)$ phase to grow in the liquid-disordered $\left(l_{d}\right)$ membrane [25,31-33]. The first $l_{0}$ domains always emerge in regions where two unilamellar membranes adhere. An example is shown in 


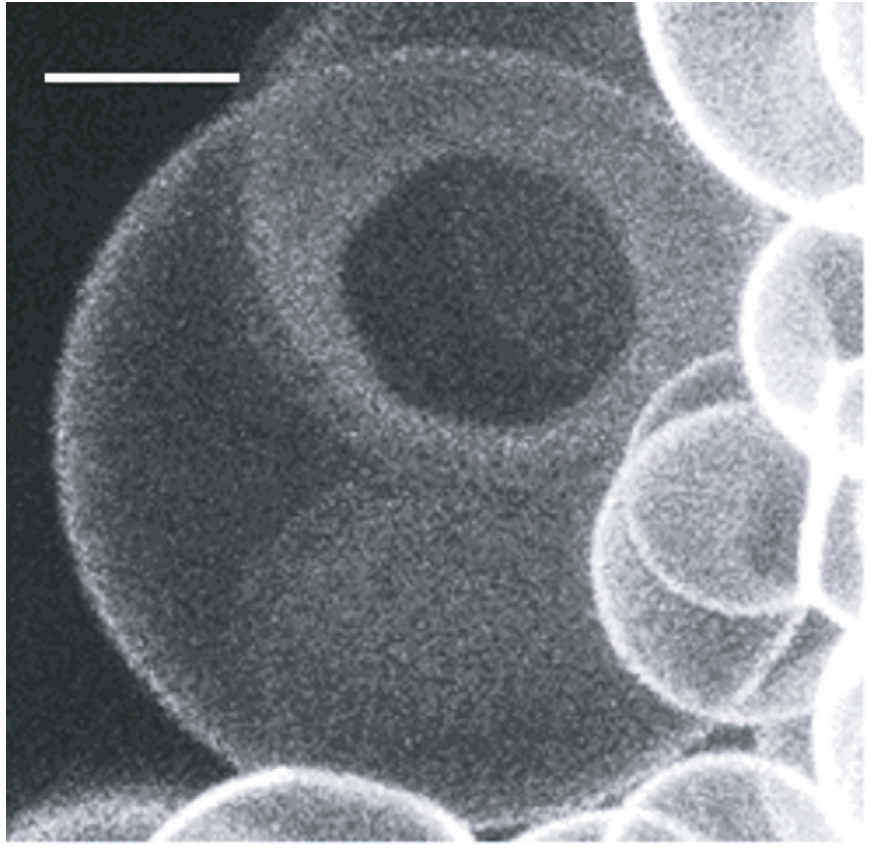

Fig. 2: A hexagonal $\mathrm{P}_{\beta^{\prime}}$ II gel domain spans adhering DLPCDSPC membranes (mixture 2). Notice that this ordered-phase domain excludes the dyes BODIPY and DiI-C-18. Observation temperature: $43^{\circ} \mathrm{C}$. Scale bar: $5 \mu \mathrm{m}$.

fig. 1, where two round $\mathrm{l}_{\mathrm{o}}$ domains appear in two disjoint contact zones between three adhering vesicles.

Analogous observations were made for the four fluid-solid mixtures 2-5. As the temperature is lowered, a transition point is reached below which domains of an ordered gel phase grow in their surrounding fluid matrix $[28,34,35]$. For the three binary mixtures $2-4$, in which the larger lipid has a tail with 18 or 20 carbon atoms, this ordered phase is $\mathrm{P}_{\beta^{\prime}}$ II [29]. We always observed this phase to nucleate and grow in registry across the two bilayers of adhering unilamellar vesicles. Figure 2 provides an illustration of an adhesion zone that has localized a $\mathrm{P}_{\beta^{\prime}}$ II domain, recognizable by its dye exclusion and characteristic hexagonal symmetry [6]. In mixture 5, in which the larger lipid (DPPC) has a tail with 16 carbon atoms, the gel domains have the structure of the more usual ripple phase $\mathrm{P}_{\beta^{\prime}}$ [30]. This corrugated and thus highly anisotropic phase forms stripe domains. Again, we found them to emerge first in adhering regions, when membrane tension was released by slightly deflating the vesicles and after we cooled slowly $\left(\sim 0.2^{\circ} \mathrm{C} / \mathrm{min}\right)$ towards the transition temperature. Figure 3 provides an illustration of the striped gel phase in a contact zone. Notably, this time domains did not grow in registry across the two adhering bilayers.

To summarize, for all five systems investigated we find that ordered domains (liquid-ordered or solid-like) first form in regions where two membranes adhere. Such consistent localization implies that bilayer adhesion stabilizes or strongly favours these ordered lipid phases.
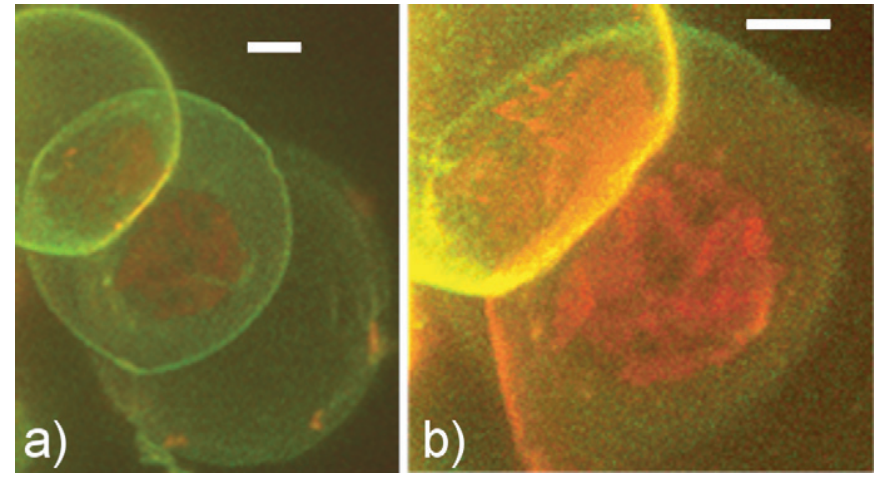

Fig. 3: (Colour on-line) $\mathrm{A}_{\beta^{\prime}}$ gel phase grows in the contact zone of two adhering vesicles of lipid mixture 5 . The overlapping stripe-like domains are not in registry across the two bilayers. Notice that these domains are bright in the dye DiI-C-18 (shown in false-colour red) and exclude the dye BODIPY (shown in false-colour green). Panel (b) shows a higher magnification of (a). Observation temperature: $30^{\circ} \mathrm{C}$. Scale bars: $5 \mu \mathrm{m}$.

Before we propose a mechanism for these observations, we first rule out a number of possible explanations.

First, given that the phenomenon is observed across three different ordered phases $\left(\mathrm{l}_{\mathrm{O}}, \mathrm{P}_{\beta^{\prime}}, \mathrm{P}_{\beta^{\prime}} \mathrm{II}\right)$, any explanation which depends on the property of a particular lipid or phase is unsatisfactory. In fact, the $\mathrm{D} X \mathrm{PC}$ lipids as well as sphingomyelin all have the same zwitterionic polar head group (phosphatidylcholine). This strengthens the argument against lipid-specific cross-bilayer coupling, as does the fact that we also observe domain formation out of registry between adhering membranes (mixture 5, fig. 3). In other words, lipids do not simply demix to increase favourable inter-bilayer interactions between chemically different head groups.

Secondly, we are confident that this is not a fluorophoreinduced artifact, because the same fluorophore (DiI-C-18) that is excluded from ordered $\mathrm{l}_{\mathrm{o}}$ and $\mathrm{P}_{\beta^{\prime}}$ II domains, figs. 1 and 2, preferentially partitions into ordered domains of $\mathrm{P}_{\beta^{\prime}}$, fig. 3 .

Thirdly, even though the vesicle curvature differs between free membranes and adhesion zones, the concomitant change in bending energy density is extremely small compared to entropic terms (we will quantify this below). The issue is subtle, though, since experiment [36] and theory [37] have shown that when the entire membrane is adherent corrugated substrates can localize $l_{0}$ domains to regions of low curvature. However, when we cooled our GUVs quickly, at $0.4^{\circ} \mathrm{C} / \mathrm{min}$ or more, $\mathrm{l}_{\mathrm{o}}$ and $\mathrm{P}_{\beta^{\prime}}$ domains nucleated, grew, and remained in the free membrane over the time of observation (up to several hour) as well as the adhering membrane, with most of the ordered phase located in the free, non-adhering membrane. Our observations are therefore not due to curvature effects alone. 
We therefore conclude that adhesion per se must underlie a very generic mechanism that leads to the localization of ordered phases which we observe at slow cooling rates.

Theoretical framework. - The explanation we propose may be summarized as follows. Lipid segregation is typically viewed as a competition between the energy gained from avoiding contacts between unlike lipids and the entropic cost resulting from demixing them. Yet, membrane undulations also contribute to this balance and thereby renormalize the transition temperature. Hence, any mechanism interfering with shape fluctuations — such as adhesion - shifts the point of demixing. We now make this idea quantitative.

Within the classical continuum description of fluid membranes [38], a free-standing, nearly flat bilayer can be conceptualized as a collection of independent harmonic oscillators - one for each undulation mode of wave vector $q$ [39]. Each of these oscillators has a spring constant $\kappa q^{4}$, where $\kappa$ is the bending stiffness. The classical free energy of such a simple one-dimensional harmonic oscillator satisfies $F / k_{\mathrm{B}} T \sim \ln (\omega)$, where $k_{\mathrm{B}} T$ is the thermal energy and $\omega \sim \kappa^{1 / 2}$ is the oscillator frequency. Stiffening the spring increases its free energy, since fluctuations become more costly. This is a purely entropic effect, as the average energy of any quadratic degree of freedom is always $\frac{1}{2} k_{\mathrm{B}} T$.

For a two-component membrane, the bending modulus $\kappa_{\mathrm{AB}}$ of the membrane made of the homogeneously mixed components (A and $\mathrm{B}$ ) will generally differ from the bending moduli $\kappa_{\mathrm{A}}$ and $\kappa_{\mathrm{B}}$ of unitary membranes made of each component $[40,41]$. To expose the physics, we neglect some of the subtleties of demixing near a liquidus and consider a 50:50 mixed membrane segregating into two equal-sized patches of pure $\mathrm{A}$ and $\mathrm{B}$. The fluctuation contribution to the free energy per area will change by $\Delta f=\frac{1}{2}\left(f_{\mathrm{A}}+f_{\mathrm{B}}\right)-f_{\mathrm{AB}}$. Summing over all modes $[42,43]$, the total free-energy difference per area is found to be

$$
\Delta f=\frac{\pi}{4} \times \frac{k_{\mathrm{B}} T}{a^{2}} \times \ln \frac{\kappa_{\mathrm{A}} \kappa_{\mathrm{B}}}{\kappa_{\mathrm{AB}}^{2}},
$$

where $a=2 \pi / q_{>}$is the smallest length for which a continuum description is meaningful, and $q_{>}$acts therefore as an "ultraviolet cut-off".

In our experiment a homogeneous fluid bilayer can demix into one A-rich disordered-fluid phase and one B-rich ordered phase, which is stiffer than the disordered fluid phase. In the simplest approximation we therefore have $\kappa_{\mathrm{AB}} \approx \kappa_{\mathrm{A}} \ll \kappa_{\mathrm{B}}=\alpha \kappa_{\mathrm{A}}$, where the stiffening factor $\alpha$ is of the order of ten for solid-like phases [44-47], and is more than two for the fluid-ordered phase $[8,9,31]$. Inserting this approximation into eq. (1) we find

$$
\Delta f \simeq \frac{\pi}{4} \times \frac{k_{\mathrm{B}} T}{a^{2}} \times \ln \alpha>0 .
$$

Undulations favour mixing, so that suppressing them tips the balance towards phase segregation. Membrane

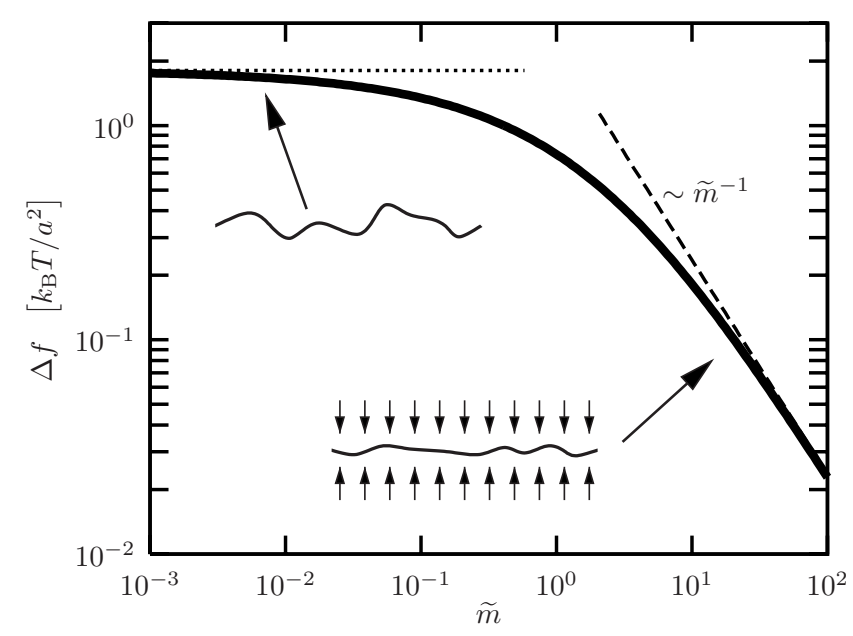

Fig. 4: Fluctuation contribution $\Delta f$ to the free energy of demixing, eq. (3), as a function of the dimensionless confinement parameter $\widetilde{m}=m / \kappa q_{>}^{4}$ for $\alpha=10$. Increasing the strength of the confinement reduces the fluctuation contribution to mixing, thus favouring phase separation.

adhesion, which generically suppresses fluctuations, may thus drive demixing.

To illustrate the point, let us model the adhesionderived confinement by a harmonic potential $\frac{1}{2} m h^{2}$, where $h(x, y)$ is the local deviation from the average bilayer plane and $m$ characterizes the "strength" of the confinement. The membrane can still be described by a set of independent harmonic oscillators, but this time with a spring constant of $\kappa q^{4}+m$. Returning to the demixed membrane consisting of two equal-sized $\mathrm{A}$ and $\mathrm{B}$ patches, but this time with confinement, $\Delta f$ from eq. (1) is replaced by the confinement-dependent fluctuation contribution to the demixing free energy

$$
\Delta f \simeq \frac{\pi}{4} \times \frac{k_{\mathrm{B}} T}{a^{2}} \times\left\{\ln \frac{\alpha+\widetilde{m}}{1+\widetilde{m}}+\mathcal{A}(\widetilde{m} / \alpha)-\mathcal{A}(\widetilde{m})\right\}
$$

where we have defined the function $\mathcal{A}(x)$ by

$$
\mathcal{A}(x)=2 \sqrt{x} \arctan \frac{1}{\sqrt{x}}
$$

and where $\widetilde{m}=m / \kappa q_{>}^{4}$ is the (dimensionless) confinement modulus. Notice that in the free limit $m \rightarrow 0$ this reduces to the simpler unconfined fluctuation expression from eq. (2). For $\alpha=10$ the function $\Delta f(\widetilde{m})$ is shown in fig. 4. As expected, with increasing $\widetilde{m}$ the fluctuation contribution to the demixing free energy vanishes, so confinement favours demixing. In consequence, adherent patches in two-component membranes ought to demix at temperatures slightly above the transition temperature for the non-adherent membrane.

How big is this effect? For a stiffening factor $\alpha=10$ undulations will contribute a demixing free energy of 
$\frac{\pi}{4} \ln (10) k_{\mathrm{B}} T \approx 1.8 k_{\mathrm{B}} T$ for a patch of area $a^{2}$. Taking $a=10 \mathrm{~nm}$, such a patch contains on the order of 300 lipids, each contributing $\ln (2) k_{\mathrm{B}} T$ mixing free energy. The undulation correction thus amounts to about $1 \%$, or $3 \mathrm{~K}$ at room temperature. This is an easily measurable effect, which becomes stronger if demixing is incomplete, as the relative importance of undulations compared to the mixing entropy increases. Under typical experimental conditions the effect will be reduced, though, because i) some fluctuations may remain after adhesion and ii) a tension in the non-adhering membrane reduces the free fluctuations. Still, we expect a measurable change in the transition temperature upon going from a unilamellar membrane to the fluctuation-damped, adhesion-confined double bilayer membrane of the same composition.

It is easy to see that in our case a macroscopic curvature change is negligible compared to the fluctuation effect. The elastic bending energy per area of a membrane with spherical curvature of radius $R$ is given by $2 \kappa / R^{2}$. Using the same notation and approximation as above, the elastic energy change upon demixing amounts to

$$
\Delta f_{\text {curvature }}=\frac{\kappa_{\mathrm{AB}}}{a^{2}} \times(\alpha-1) \times\left(\frac{a}{R}\right)^{2} .
$$

Taking $\kappa_{\mathrm{AB}} \simeq 20 k_{\mathrm{B}} T, \alpha=10, a=10 \mathrm{~nm}$ and $R=10 \mu \mathrm{m}$, we find a change of $1.8 \times 10^{-4} k_{\mathrm{B}} T$ for a patch of area $a^{2}$, which is four orders of magnitude less than the fluctuation contribution. Stated differently, both effects become equal in magnitude for $R \simeq 100 \mathrm{~nm}$, which is significantly smaller than the curvature seen in our GUVs.

A full theory of non-specific adhesion between two fluctuating bilayers should also consider a finite tension. Its inclusion into the above model is conceptually straightforward, but leads to cumbersome equations. For this reason we have restricted ourselves to the tension-free scenario, which allows us to calculate the consequences of fluctuation suppression by confinement analytically, thereby illustrating the basic mechanism as clearly as possible.

Conclusion. - In their pioneering theoretical work on how undulations affect membrane interactions, Evans and Parsegian wrote that "stable adhesion is predicted to be promoted by membrane rigidification" [48]. Our observations show that for mixed membranes the reverse also holds: adhesion may promote membrane rigidification. In our case, rigidification is due to demixing and the localization of the more rigid phase in the adhesion zone. The end result is that adhesion promotes demixing in a system that is already close to an ordering transition.

Several recent observations on promoted demixing in model membranes are in accord with with the mechanism we describe in this paper [49-51]. Perhaps most straightforwardly, our model suggests a mechanism by which phase separation producing stiffer, less-fluctuating domains in one leaflet of an asymmetric bilayer could promote phase separation in the second leaflet, even when that second lipid composition does not favour phase separation [51].

Real biomembranes contain complex mixtures of lipids and proteins [14], and lateral heterogeneities [1,2,13] play essential roles in key processes such as endocytosis [52], exocytosis [53], intracellular trafficking [54], and pathogen infection [55]. Our observations and theoretical modelling suggest a novel mechanism for generating and controlling such heterogeneities that is driven by the lipids themselves rather than membrane-bound proteins. The close approach of a cell to any biological or non-biological surface may lead to local phase separation in the membranes at the contact region, provided that external conditions (temperature, $p \mathrm{H}$, etc.) already take the membranes close to demixing. This suggestion is particularly intriguing in view of the recent proposal that critical composition fluctuations may be a source of lipid rafts [12].

Note finally that adhesion-promoted phase separation can easily be turned into a means of functional control. If initial adhesion driven by a few specific linker molecules abates fluctuations sufficiently strongly to create an $l_{0}$ domain in the adhesion zone, and if this phase also preferentially solubilizes these linkers (as it is known to be true for activated integrins [56]), our generic demixing scenario effectively acts as a non-linear switch to amplify adhesion points of sufficient initial stability.

$$
* * *
$$

We thank P. Bassereau, M. E. Cates, P. S. ClegG, J. Israelachvili, P. Janmey, F. C. MacKintosh, M. Rappolt, M. Shipston, and N. VAN DER VEgT for discussions, A. Downie and P. A. BeAles for constructing the electroformation chamber, and J. ARLT and A. GARRIE for technical support. VDG was funded by the EPSRC Grant GR/S10377 (Edinburgh Soft Matter and Statistical Physics Program Grant). MD acknowledges financial support by the DFG through grants De775/1-2 and De775/1-3.

\section{REFERENCES}

[1] Simons K. and Ikonen E., Nature, 387 (1997) 569.

[2] Brown D. A. and London E., J. Membr. Biol., 164 (1998) 103

[3] Beales P. A., Gordon V. D., Zhao Z., EgelhaAf S. U. and Poon W. C. K., J. Phys.: Condens. Matter, 17 (2005) S3341.

[4] Korlach J., Schwille P., Webb W. W. and Feigenson G. W., Proc. Natl. Acad. Sci. U.S.A., 96 (1999) 8461.

[5] Bagatolli L. A. and Gratton E., Biophys. J., 78 (2000) 434.

[6] Gordon V. D., Beales P. A., Shearman G. C., Zhao Z., Seddon J. M., EgelhaAf S. U. and Poon W. C. K., submitted to Phys. Rev. E. 
[7] Veatch S. L. and Keller S. L., Phys. Rev. Lett., 89 (2002) 268101.

[8] Baumgart T., Hess S. T. and Webb W. W., Nature, 425 (2003) 821.

[9] Baumgart T., Das S., Webb W. W. and Jenkins J. T., Biophys. J., 89 (2005) 1067.

[10] Bacia K., Schwille P. and Kurzchalia T., Proc. Natl. Acad. Sci. U.S.A., 102 (2005) 3272.

[11] Cicuta P., Keller S. L. and Veatch S. L., J. Phys. Chem. B, 111 (2007) 3328.

[12] Veatch S. L., Soubias O., Keller S. L. and Gawrisch K., Proc. Natl. Acad. Sci. U.S.A., 104 (2007) 17650.

[13] Engelman D. M., Nature, 438 (2005) 578.

[14] Alberts B., Johnson A., Lewis J., Raff M., Roberts K. and Walter P., Molecular Biology of the Cell, 4th edition (Garland Science, New York) 2002.

[15] Jamora C. and Fuchs E., Nat. Cell Biol., 4 (2002) 101.

[16] El-Ali J., Sorger P. K. and Jensen K. F., Nature, 442 (2006) 403.

[17] Lee K.-H., Holdorf A. D., Dultin M. L., Chan A. C., Allen P. M. and ShaW A. S., Science, 245 (2002) 1539 .

[18] Sackmann E. and Bruinsma R. F., Chem Phys Chem, 3 (2002) 262.

[19] Boulbitch A., Guttenberg Z. and Sackmann E., Biophys. J., 81 (2001) 2743.

[20] Marchi-Artzner V., Lorz B., Gosse C., Jullien L., Merkel R., Kessler H. and Sackmann E., Langmuir, 19 (2003) 835.

[21] Smith A. S., Lorz B. G., Seifert U. and Sackmann E., Biophys. J., 90 (2006) 1064.

[22] Shenoy V. B. and Freund L. B., Proc. Natl. Acad. Sci. U.S.A., 102 (2005) 3213.

[23] Qi S. Y., Groves J. T. and Chakraborty A. K., Proc. Natl. Acad. Sci. U.S.A., 98 (2001) 6548.

[24] Weikl T. R. and Lipowsky R., Biophys. J., 87 (2004) 3665.

[25] Veatch S. L. and Keller S. L., Biophys. J., 84 (2003) 725.

[26] Angelova M. I. and Dimitrov D. S., Faraday Discuss. Chem. Soc., 81 (1986) 303.

[27] Manley S. and Gordon V. D., Curr. Protoc. Cell Biol. (2008) Unit 24.3.

[28] Gordon V. D., Beales P. A., Zhao Z., Blake C., Mackintosh F. C., Olmsted P. D., Cates M. E., EgelhaAf S. U. and Poon W. C. K., J. Phys.: Condens. Matter, 18 (2006) L415.

[29] Pressl K., Jørgensen K. and Laggner P., Biochim. Biophys. Acta, 1325 (1997) 1.

[30] Hentschel M. P. and Rustichelli F., Phys. Rev. Lett., 66 (1991) 903.
[31] Roux A., Cuvelier D., Nassoy P., Prost J., Bassereau P. and Goud B., EMBO J., 24 (2005) 1537.

[32] Ayuyan A. G. and Cohen F. S., Biophys. J., 91 (2006) 2172.

[33] Yuan J., Hira S. M., Strouse G. F. and Hirst L. S., J. Am. Chem. Soc., 130 (2008) 2067.

[34] Luzzati V. and Tardieu A., Annu. Rev. Phys. Chem., 25 (1974) 79.

[35] Koynova R. and Caffrey M., Biochim. Biophys. Acta, 1376 (1998) 91.

[36] Parthasarathy R., Yu C. and Groves J. T., Langmuir, 22 (2006) 5095.

[37] Różycki B., Weikl T. R. and Lipowsky R., Phys. Rev. Lett., 100 (2008) 098103.

[38] Helfrich W., Z. Naturforsch. C, 28 (1973) 693.

[39] BoAl D., Mechanics of the Cell (Cambridge University Press, Cambridge) 2002.

[40] Szleifer I., Kramer D., Ben-Shaul A., Roux D. and Gelbart W. M., Phys. Rev. Lett., 60 (1988) 1966.

[41] Imparato A., Shillcock J. C. and Lipowsky R., Europhys. Lett., 69 (2005) 650.

[42] Brochard F., de Gennes P. G. and Pfeuty P., J. Phys. (Paris), 37 (1976) 1099.

[43] Morse D. C. and Milner S. T., Phys. Rev. E, 52 (1995) 5918.

[44] Dimova R., Pouligny B. and Dietrich C., Biophys. J., 79 (2000) 340.

[45] Lee C.-H., Lin W.-C. and Wang J., Phys. Rev. E, 64 (2001) 020901.

[46] Pècrèaux J., Döbereiner H.-G., Prost J., JoAnny J.-F. and Bassereau P., Eur. Phys. J. E, 13 (2004) 277.

[47] Daillant J., Bellet-Amalric E., Braslau A., Charitat T., Fragneto G., Graner F., Mora S., Rieutord F. and Stidder B., Proc. Natl. Acad. Sci. U.S.A., 102 (2005) 11639.

[48] Evans E. A. and Parsegian V. A., Proc. Natl. Acad. Sci. U.S.A., 83 (1986) 7132.

[49] Liu A. P. and Fletcher D. A., Biophys. J., 91 (2006) 4064.

[50] Hammond A. T., Heberle F. A., Baumgart T., Holowka D., Baird B. and Feigenson G. W., Proc. Natl. Acad. Sci. U.S.A., 102 (2005) 6320.

[51] Collins M. D. and Keller S. L., Proc. Natl. Acad. Sci. U.S.A., 105 (2008) 124.

[52] Parton R. G. and Richards A. A., Traffic, 4 (2003) 724.

[53] Salaün C., James D. J. and Chamberlain L. H., Traffic, 5 (2004) 255.

[54] Helms J. B. and Zurzolo C., Traffic, 5 (2004) 247.

[55] Marsh M. and Helenius A., Cell, 124 (2006) 729.

[56] Leitinger B. and Hogg N., J. Cell Sci., 115 (2002) 963. 